\title{
Carbon xerogel supported noble metal catalysts for fine chemical applications
}

\author{
B.F. Machado ${ }^{a}$, H.T. Gomes ${ }^{a, b}$, Ph. Serpc, Ph. Kalckc, J.L. Figueiredo ${ }^{a}$, J.L. Fariaa,* \\ a Laboratório de Catálise e Materiais, Departamento de Engenharia Química, Faculdade de Engenharia, \\ Universidade do Porto, 4200-465 Porto, Portugal \\ b Departamento de Tecnologia Química e Biológica, Escola Superior de Tecnologia e de Gestão, Instituto \\ Politécnico de Bragança, 5300-857 Bragança, Portugal \\ c Laboratoire de Chimie de Coordination - CNRS UPR8241, Ecole Nationale Supérieure d'Ingénieurs en \\ Arts Chimiques Et Technologiques, Toulouse, 31077 France \\ *Corresponding author: fax: +351 22508 1449, jlfaria@fe.up.pt
}

\begin{abstract}
Carbon xerogel, a mesoporous material, was produced by polycondensation of resorcinol and formaldehyde. A concentrated nitric acid solution was subsequently used to introduce high amounts of oxygenated groups on the surface. The carbon xerogel served as a support for Pt, Ir and $\mathrm{Ru}$ monometallic catalysts by using organometallic precursors. The catalysts were tested in the liquid-phase selective hydrogenation of cinnamaldehyde to cinnamyl alcohol. The introduction of surface groups was important to increase metal dispersion but proved to limit selectivity towards the unsaturated alcohol. After a thermal treatment at $973 \mathrm{~K}$ the catalysts showed excellent thermal stability and a narrow metal size distribution. Regarding the catalytic results measured at 50\% conversion, Pt catalysts exhibited the highest selectivity to cinnamyl alcohol (73 \%) followed by Ir with $65 \%$ and finally Ru with only $32 \%$.
\end{abstract}

Keywords: carbon xerogel; surface chemistry; selective hydrogenation; cinnamaldehyde

\section{Introduction}

Efficiency and environmental impact are pushing the interest in the use of heterogeneous catalysts for the synthesis of fine chemicals. Indeed, heterogeneous catalytic processes are always easier to handle than the homogeneous ones and lead to lower amounts of waste products, thus lower risk for the environment. Research focused on chemo- and regioselective catalytic hydrogenation of unsaturated compounds to produce fine chemicals is rapidly growing. In the past years considerable efforts have been devoted developing catalytic systems able to perform selective hydrogenation of the carbonyl function in $\alpha, \beta$-unsaturated aldehydes, to form the corresponding alcohols with a high yield, thus avoiding the use of toxic reducing agents such as metal hydrides, commonly applied in preparative organic chemistry.

The unsaturated alcohols are important intermediates in the organic synthesis of fine chemicals for several industries such as flavor, fragrance, pharmaceutical and agrochemical. Unfortunately, high selectivity towards these alcohols is difficult to achieve, since 
thermodynamics favors the hydrogenation of the $\mathrm{C}=\mathrm{C}$ over the $\mathrm{C}=\mathrm{O}$ bond by about $35 \mathrm{~kJ} \mathrm{~mol}^{-1}$ and the reactivity of the olefinic bond is higher than that the carbonyl. To increase selectivity, these thermodynamic and kinetic constraints must be reduced or even removed.

It has been found that selectivity towards the allylic alcohol is highly dependent on the nature of the precious metal used. Metals such as Pt, Os, Ir, Pd, Rh and Ru among others have been studied, leading to differences in activity and selectivity. Gallezot and Richard [1] reported that unpromoted Ir and Os catalysts are considered to be rather selective for unsaturated alcohol formation while $\mathrm{Pt}, \mathrm{Ru}$ and $\mathrm{Co}$ are moderately selective and $\mathrm{Pd}, \mathrm{Rh}$ and $\mathrm{Ni}$ are nonselective. Because the support plays an important role in catalyst design, in order to optimize the dichotomy between metal and support, the use of a material whose properties can be finely tuned is highly desirable. Carbon xerogels (CX) are a relatively new member of the carbon family, and the possibility of adjusting the surface area and pore width to a certain extent, among other textural properties, makes its use very attractive.

In this work we have studied the effect of high temperature activation of CX supported noble metal catalysts on their performances using the hydrogenation of cinnamaldehyde as model reaction.

\section{Experimental}

An aqueous organic gel was prepared by polycondensation of resorcinol with formaldehyde (1:2), adapting the procedure described by Job et al. [2]. Accordingly, $56.57 \mathrm{~g}$ of resorcinol were added to $113 \mathrm{~mL}$ of deionised water in a glass flask. After complete dissolution, $82 \mathrm{~mL}$ of formaldehyde solution were also added. The initial $\mathrm{pH}$ was set at 6.0 by adding sodium hydroxide solution ( $2 \mathrm{M})$ dropwise under continuous stirring. The gelation step was allowed to proceed at $358 \mathrm{~K}$ during 3 days, after which the gel displayed a dark red colour. At this point, the consistency of the material allowed the sample to be shaped as desired (ground to small particles $c a .0 .2 \mathrm{~mm}$ ). The gel was further dried in an oven from $333 \mathrm{~K}$ to $423 \mathrm{~K}$ during

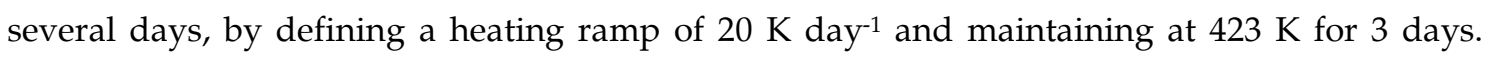
After drying, the gel was pyrolyzed at $1073 \mathrm{~K}$ under a constant nitrogen flow $\left(100 \mathrm{~mL} \mathrm{~min}{ }^{-1}\right)$ in a vertical tubular furnace. The gel was then treated in nitric acid solution $(7 \mathrm{M})$ to create oxygen surface groups, washed with water until neutrality of the rinsing waters and dried in oven for 2 days at $413 \mathrm{~K}$.

Monometallic catalysts supported on $\mathrm{HNO}_{3}$-oxidized carbon xerogel containing $1 \mathrm{wt}$. \% of platinum, iridium and ruthenium were prepared by wet impregnation under inert atmosphere using $\left[\mathrm{Pt}\left(\mathrm{CH}_{3}\right)_{2}(\mathrm{COD})\right], \quad\left[\left(\operatorname{Ir}\left(\mu-\mathrm{SC}\left(\mathrm{CH}_{3}\right)_{3}\right)(\mathrm{CO})_{2}\right)_{2}\right]$ and $[\mathrm{Ru}(\mathrm{COD})(\mathrm{COT})]$ as organometallic precursors, respectively. After 1 day under vigorous stirring the catalysts were filtered and dried overnight in the oven ( $393 \mathrm{~K})$. Prior to reaction the catalysts were calcined in 
nitrogen and reduced with hydrogen (Pt/CX and $\mathrm{Ru} / \mathrm{CX}$ at $623 \mathrm{~K}$ and $\mathrm{Ir} / \mathrm{CX}$ at $773 \mathrm{~K}$ ). In the case of the Ir catalyst it was necessary to remove the sulfur contained in the precursor, therefore we used a higher temperature [3]. A post-reduction thermal treatment (PRT) under nitrogen at $973 \mathrm{~K}$ was also performed (Pt/CX973, Ir/CX973 and Ru/CX973) to remove excess surface groups.

Temperature programmed desorption (TPD) spectra of $\mathrm{CO}$ and $\mathrm{CO}_{2}$ were obtained with a fully automated AMI-200 Catalyst Characterization Instrument (Altamira Instruments), equipped with a quadrupole mass spectrometer (Dymaxion $200 \mathrm{amu}$, Ametek). In a typical experiment, $0.1 \mathrm{~g}$ are placed in a U-shaped quartz tube located inside an electrical furnace and heated up to $1373 \mathrm{~K}$ at $5 \mathrm{~K} \mathrm{~min}^{-1}$ using a constant flow rate of helium $\left(25 \mathrm{~cm}^{3} \mathrm{~min}^{-1}\right)$. For quantification of the amounts of $\mathrm{CO}$ and $\mathrm{CO}_{2}$ desorbed from the sample, calibration of these gases was carried out at the end of the analysis. $\mathrm{H}_{2}$ chemisorption measurements were performed using the same TPD apparatus. The metal dispersion was determined at room temperature in a U-shaped tubular quartz reactor after a thermal treatment to remove contaminant species from the catalyst surface. Pulses of $\mathrm{H}_{2}$ were injected through a calibrated loop into the sample at regular time intervals until the area of the peaks became constant. The amount of $\mathrm{H}_{2}$ chemisorbed was calculated from the area of the resultant peaks. Nitrogen adsorption-desorption isotherms at $77 \mathrm{~K}$ were obtained using a Coulter Omnisorp 100CX apparatus. BET equation and t-method (carbon standard isotherm [4]) were used to determine specific BET $\left(\mathrm{S}_{\mathrm{BET}}\right)$ and mesoporous $\left(\mathrm{S}_{\mathrm{MES}}\right)$ surface areas and micropore volume $\left(\mathrm{V}_{\mathrm{MIC}}\right)$. Surface analysis for topographical characterization was carried out by scanning electron microscopy (SEM) with a JEOL JSM-6301F (15 keV) electron microscope equipped with an OXFORD INCA ENERGY 350 energy dispersive X-ray spectroscopy (EDS) system. The sample powders were mounted on a double-sided adhesive tape and observed at different magnifications under two different detection modes, secondary and back-scattered electrons.

Liquid phase hydrogenation of cinnamaldehyde was carried out in a $100 \mathrm{~mL}$ stainless steel autoclave at $363 \mathrm{~K}$ and 10 bar. The reaction mixture contained heptane, cinnamaldehyde, decane (as internal standard for GC) and the catalyst. Small aliquots of the reaction mixture were taken throughout the reaction to perform quantitative analysis (conversion and product selectivity) by gas chromatography (DANI GC-1000 equipped with a WCOT Fused Silica $30 \mathrm{~m}$, $0.32 \mathrm{~mm}$ i.d., CP-Sil 8 CB low bleed/MS $1 \mu \mathrm{m}$ film coating column).

\section{Results and Discussion}

TPD experiments performed on CX before and after nitric acid activation (Figure 1) clearly show the difference in the amount of surface groups present in both samples. Upon oxidation, carbon materials often develop large amounts of surface groups, namely carboxylic 
acids (released in TPD at ca. $573 \mathrm{~K}$ ), anhydrides ( $800 \mathrm{~K})$, phenols (950 K), ethers (973 K), lactones (1000 K) carbonyls and quinones (1100 K) [5] depending on the type of treatment performed.
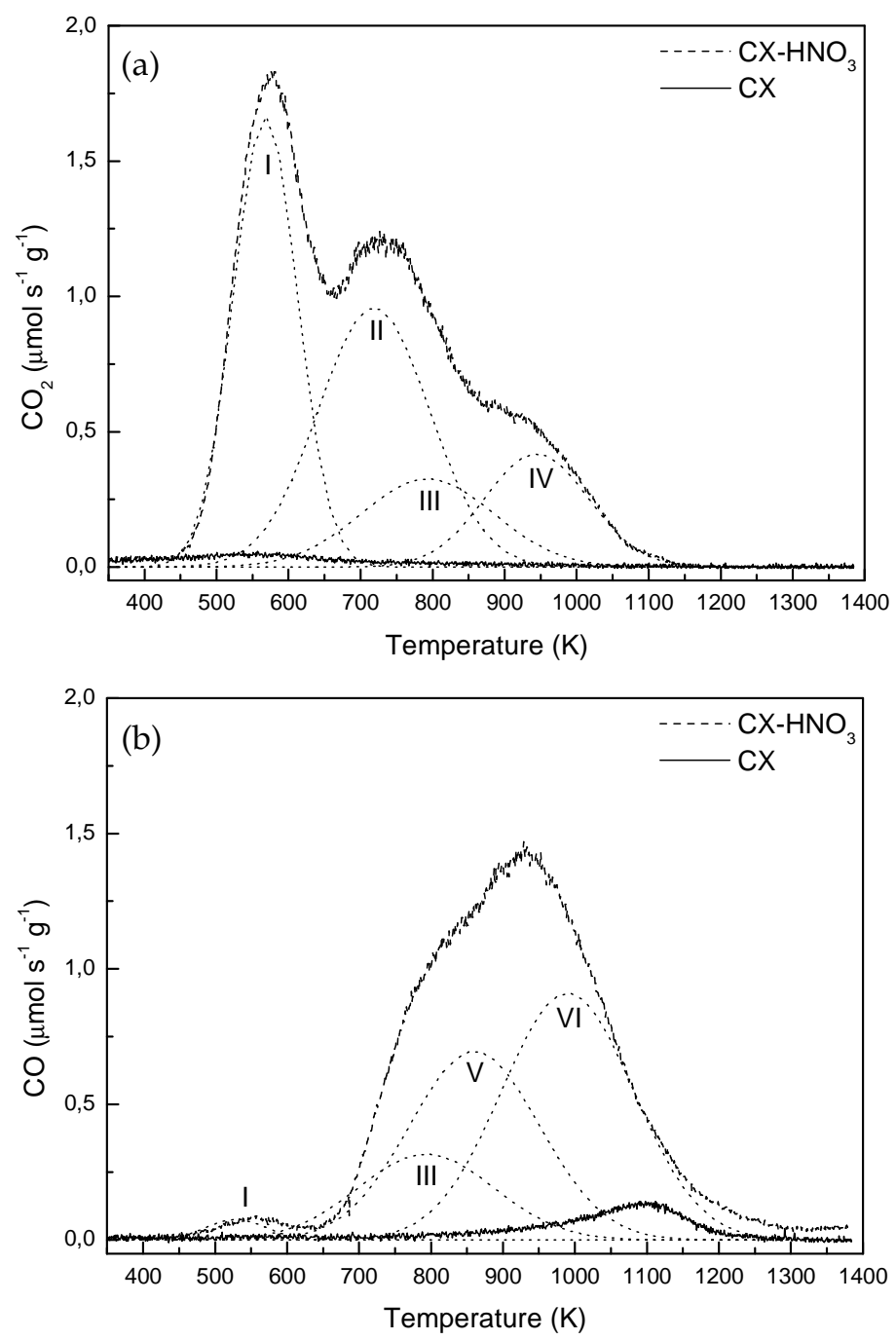

Figure 1. TPD spectra of $\mathrm{CX}$ before and after $\mathrm{HNO}_{3}$ treatment (a) $\mathrm{CO}_{2}$ and (b) $\mathrm{CO}$. See text for the meaning of roman numerals

TPD deconvolution for surface group identification is a common technique within our group and details regarding curve fitting can be found elsewhere [5,6]. Following the described procedures we were able to identify high amounts of carboxylic acid groups (I and II in Figure 1), phenols (V) and carbonyl/quinones (VI), and lower amounts of anhydrides (III) and lactones (IV) for $\mathrm{CX}-\mathrm{HNO}_{3}$. No deconvolution was carried out for untreated $\mathrm{CX}$ sample due to the very low amounts of $\mathrm{CO}$ and $\mathrm{CO}_{2}$ desorbed. An indication of the material surface acidity can be provided by the $\mathrm{CO} / \mathrm{CO}_{2}$ ratio, since most acidic groups are released as $\mathrm{CO}_{2}$, while the less acidic and basic groups release CO. A strong increase in acidity was observed upon nitric acid activation since $\mathrm{CO} / \mathrm{CO}_{2}$ decreased from $1.9(391 / 205)$ to $0.9(5468 / 6281)$. 
To study the effect of the liquid-phase activation over the texture of the material, $\mathrm{N}_{2}$ adsorption-desorption isotherms at $77 \mathrm{~K}$ were performed. Both samples produced type IV adsorption isotherms (Figure 2) with $\mathrm{H} 1$ hysteresis loops characteristic of mesoporous materials [7]. The amounts of adsorbed nitrogen were, however, very different as a drastic decrease was observed for $\mathrm{CX}-\mathrm{HNO}_{3}$. The reason why this material underwent such a strong surface area reduction has to do with the severity of the acid treatment which caused the porous structure to practically disappear as shown by the calculated specific surface area. The textural properties of both materials are summarized in Table 1.

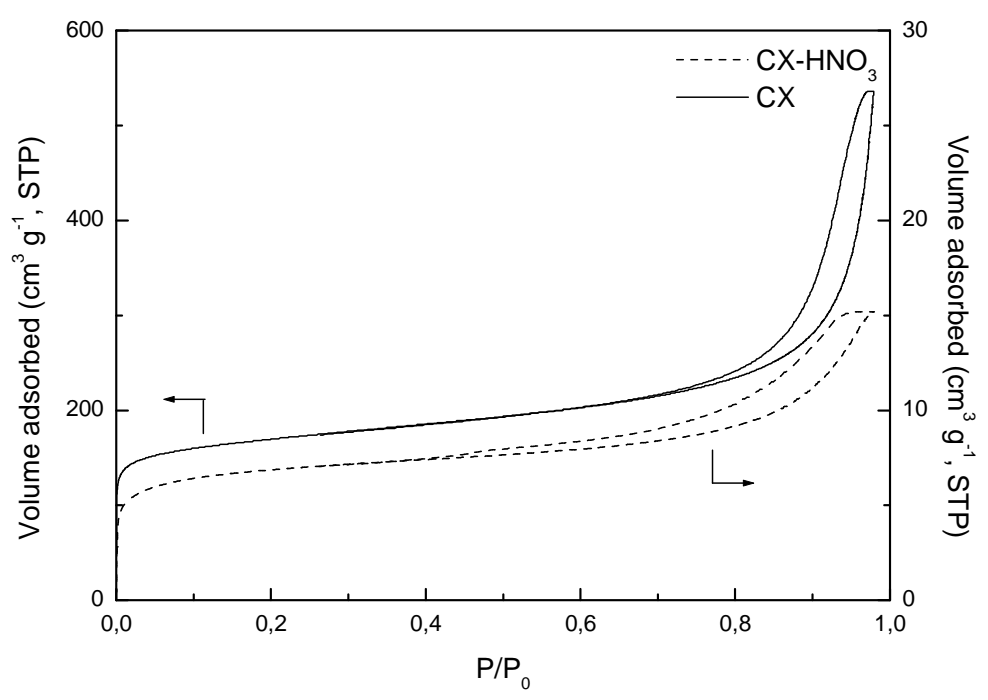

Figure 2. Nitrogen adsorption-desorption isotherms at $77 \mathrm{~K}$

SEM analysis was done in order to check why the porous structure was severely compromised. A typically high porous structure can be observed in Figure 3(a), whilst a much smoother arrangement of the surface is present in Figure $3(b)$, confirming the collapse of the structure. Comparing this new material against other carbon materials, namely single- and multi-walled nanotubes or nanofibers, we observe that the xerogel is not so stable under strong liquid-phase oxidative conditions as those, where a high degree of functionalization can be achieved without damaging the main structure. For this reason, future activations should be performed under milder acidic conditions in order to preserve the carbon xerogel pore structure.

Table 1. Textural properties of $C X$ before and after nitric acid treatment

\begin{tabular}{lccc}
\hline Sample & $\begin{array}{c}\mathbf{S}_{\text {BET }} \\
\left(\mathbf{m}^{2} \mathbf{g}^{-1}\right)\end{array}$ & $\begin{array}{c}\mathbf{S}_{\text {MES }} \\
\left(\mathbf{m}^{2} \mathbf{~} \mathbf{- 1}\right)\end{array}$ & $\begin{array}{c}\mathbf{V}_{\text {MIC }} \\
\left(\mathbf{c m}^{3} \mathbf{g}^{-1}\right)\end{array}$ \\
\hline $\mathrm{CX}$ & 627 & 180 & 0.18 \\
$\mathrm{CX}-\mathrm{HNO}_{3}$ & 20 & 6 & 0.01 \\
\hline
\end{tabular}



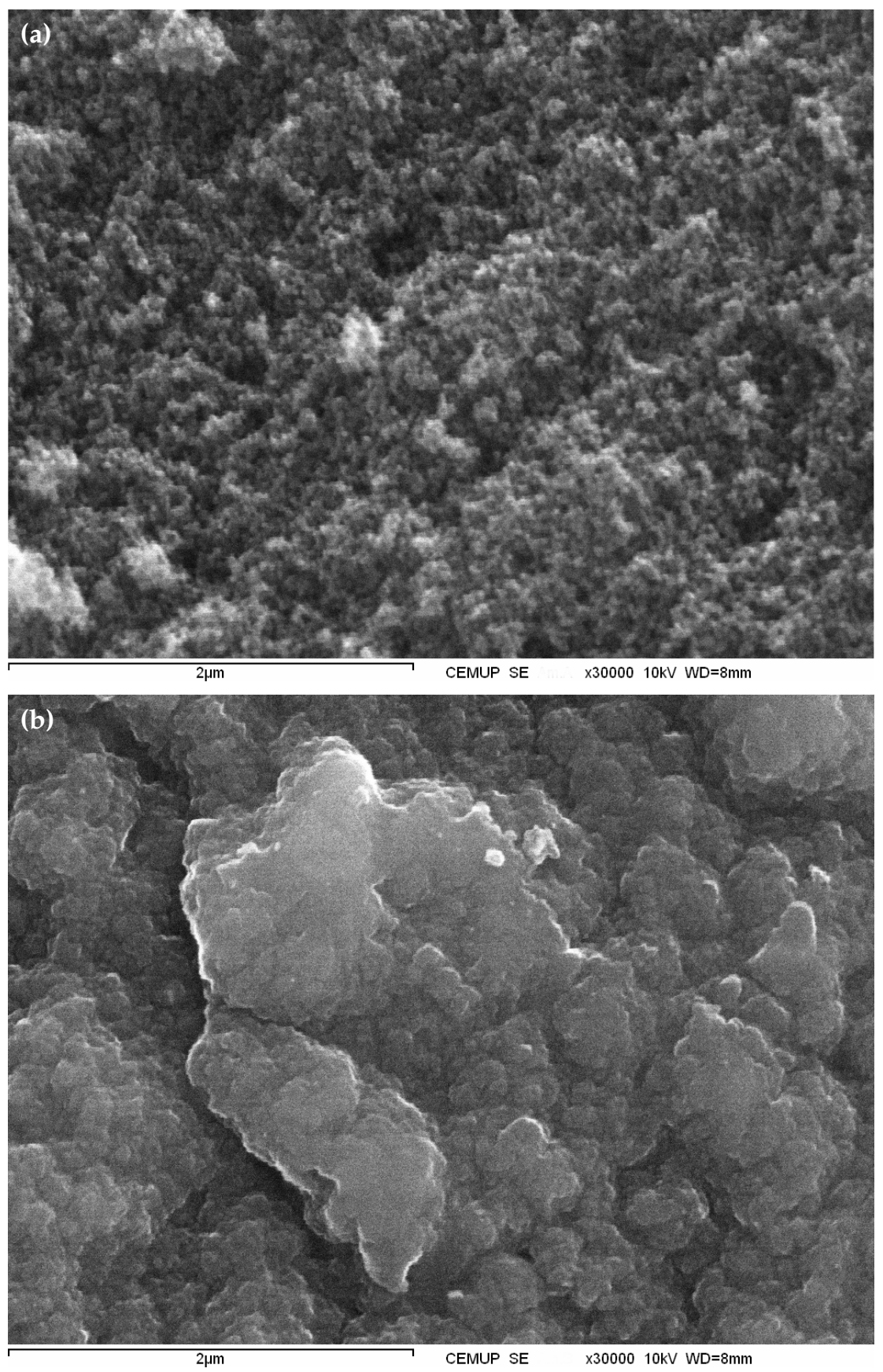

Figure 3. SEM micrographs of (a) $\mathrm{CX}$ and (b) $\mathrm{CX}-\mathrm{HNO}_{3}$

The mean metal particle size was determined from $\mathrm{H}_{2}$ chemisorption measurements, showing Pt particles around 2-3 nm, Ir with 1.5-2 nm and 1-1.5 nm in case of Ru. These results will need further confirmation by TEM analysis. This means that even with a severely decreased surface area it was possible to produce highly dispersed catalysts. The reason has to do with the remaining oxygenated surface groups, which act as anchoring sites for the metal particles during the deposition process. The strength of this bond allowed the catalysts to be treated at $973 \mathrm{~K}$ without any signs of particle sintering. Hence, it is possible to prepare highly dispersed and thermally stable catalysts by treating the surface accordingly. 
The main reaction scheme for the selective hydrogenation of cinnamaldehyde (CAL) is shown in Figure 4. The desired but thermodynamically unfavoured pathway for the preferential hydrogenation of the carbonyl group, and thus for the production of cinnamyl alcohol (COL), is evidenced in the box. Normally this route would require preferential adsorption of the $\mathrm{C}=\mathrm{O}$ group. One parallel route involves the reduction of the olefinic $\mathrm{C}=\mathrm{C}$ bond leading to hydrocinnamaldehyde (HCAL) molecule. Finally, both COL and HCAL can be further hydrogenated to produce the fully saturated alcohol, hydrocinnamyl alcohol (HCOL).

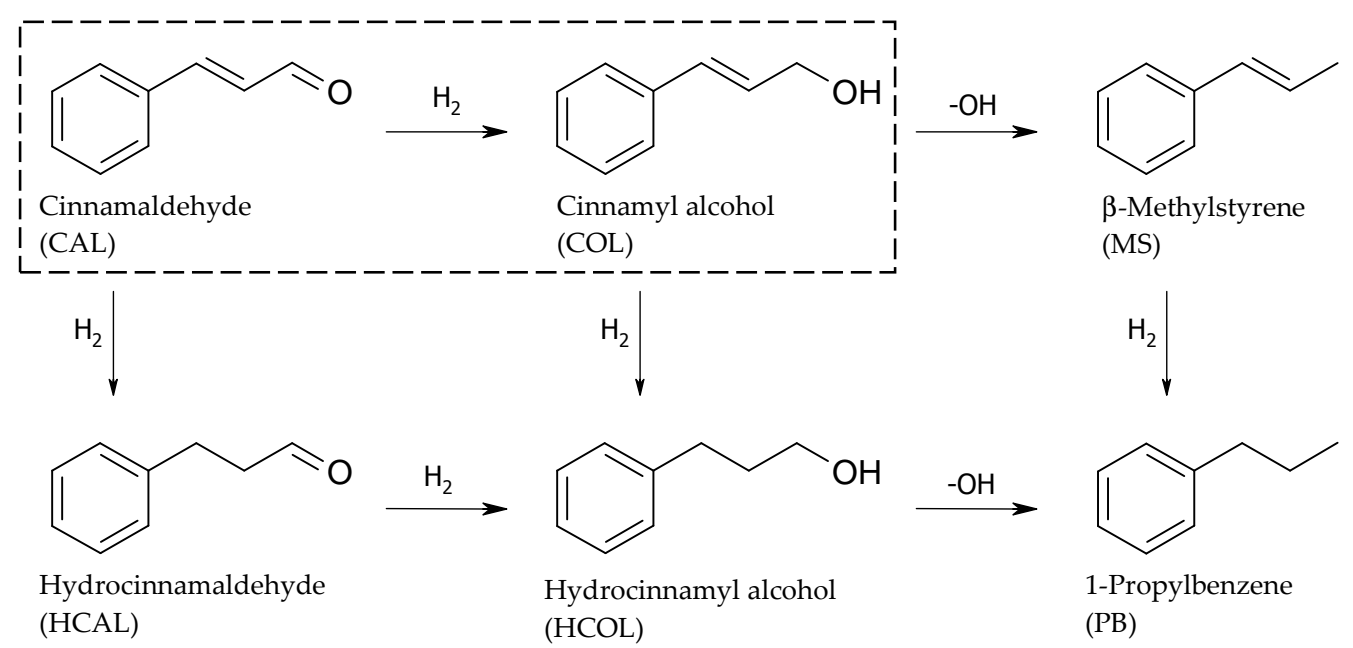

Figure 4. Pathway observed in the selective hydrogenation of cinnamaldehyde

A number of side-products that involve loss of the hydroxyl group were also detected regardless of the metal. In this case, a strong adsorption of the -OH group over the metal could help to explain their appearance. A consequence of this strong adsorption could be the poisoning of metallic sites. Catalyst reuse tests will be performed to evaluate the degree of activity and selectivity reduction. The catalytic results are gathered in Table 2.

Table 2. Catalytic results obtained in the liquid-phase hydrogenation of cinnamaldehyde with the CX supported catalysts (at $50 \%$ conversion)

\begin{tabular}{lcccc}
\hline Catalyst & $\mathbf{k}_{\mathrm{APP}}\left(\mathbf{g}_{\text {sol }} \mathbf{g}_{\mathrm{Pt}^{-1}} \mathbf{s}^{-\mathbf{1}}\right)$ & $\mathbf{S}_{\mathrm{COL}} \mathbf{( \% )}$ & $\mathbf{S}_{\mathrm{HCAL}} \mathbf{( \% )}$ & $\mathbf{S}_{\mathrm{HCOL}} \mathbf{( \% )}$ \\
\hline $\mathrm{Pt} / \mathrm{CX}$ & 4.22 & 20 & 39 & 18 \\
$\mathrm{Pt} / \mathrm{CX973}$ & 5.24 & 73 & 7 & 6 \\
$\mathrm{Ir} / \mathrm{CX}$ & 7.98 & 57 & 20 & 9 \\
$\mathrm{Ir} / \mathrm{CX} 973$ & 10.7 & 65 & 20 & 8 \\
$\mathrm{Ru} / \mathrm{CX}$ & 14.3 & 5 & 65 & 8 \\
$\mathrm{Ru} / \mathrm{CX} 973$ & 7.18 & 32 & 42 & 14 \\
\hline
\end{tabular}

CX supported catalysts exhibited enhanced selectivity after PRT at $973 \mathrm{~K}$. This treatment allowed for a better control of the catalyst surface by removing unwanted oxygen containing groups, like carboxylic acids and anhydrides. In all cases a significant improvement could be observed in the production of COL. The most outstanding result was established with $\mathrm{Pt}$ where a complete shift in HCAL to COL selectivity was noticed (Figure 5). 

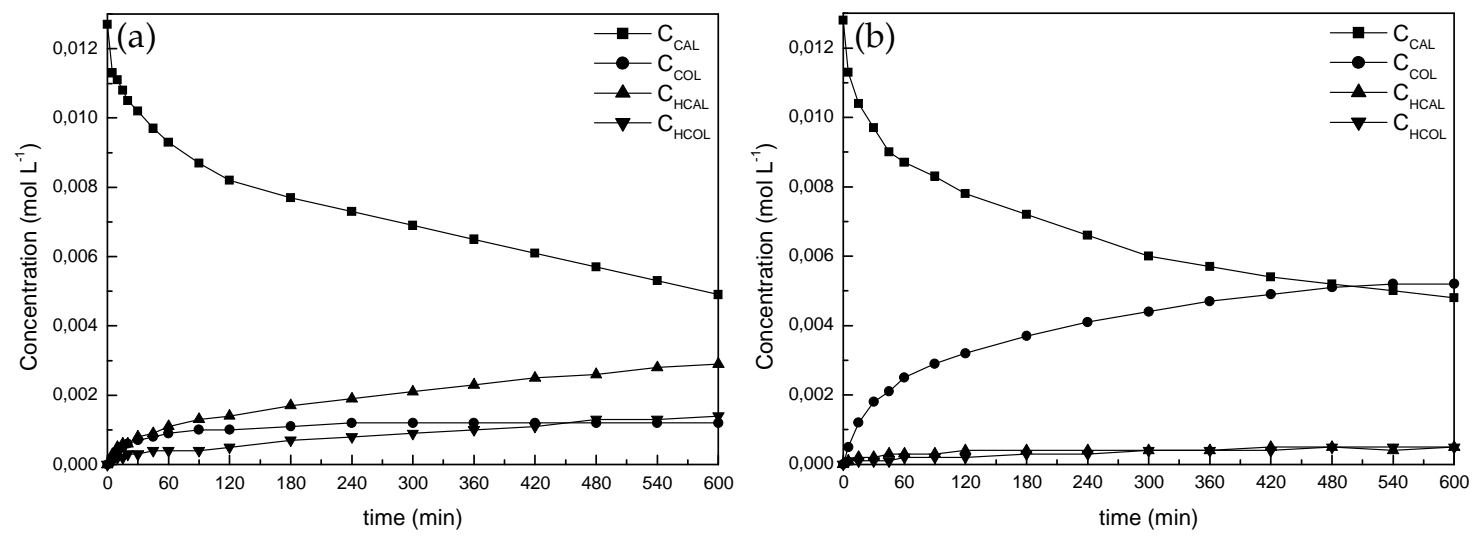

Figure 5. Concentration vs. time curves for (a) Pt/CX and (b) Pt/CX973

The best performance without PRT was observed with the Ir catalyst and this can be explained in terms of a higher processing temperature during the calcination step (necessary to remove sulfur), allowing for a partial removal of the surface groups, especially those acidic. For this reason, the enhancement observed after PRT was not as pronounced as that observed for Pt. $\mathrm{Ru}$ catalysts were, in spite of being very active, very selective towards the normally undesired HCAL (selectivity of $65 \%$ at $50 \%$ conversion).

\section{Conclusions}

The catalysts prepared from platinum, iridium and ruthenium organometallic precursors, deposited on nitric acid-oxidized carbon xerogel, presented excellent thermal stability and a narrow metal size distribution. The liquid-phase hydrogenation of cinnamaldehyde was much influenced by the thermal treatment at $973 \mathrm{~K}$, pushing the selectivity towards cinnamyl alcohol. This was attributed to preferential adsorption of $\mathrm{C}=\mathrm{O}$ after surface groups removal from $\mathrm{Pt}$ and Ir catalysts.

\section{References}

[1] P. Gallezot and D. Richard; Catal. Rev.-Sci. Eng., 40 (1998) 81.

[2] N. Job, R. Pirard, J. Marien and J.P. Pirard; Carbon, 42 (2004) 619.

[3] P. Serp, R. Feurer, P. Kalck, H. Gomes, J.L. Faria and J.L. Figueiredo; Chem. Vap. Dep., 7 (2001) 59.

[4] F. Rodriguez-Reinoso, J.M. Martin-Martinez, C. Prado-Burguete and B. McEnaney; J. Phys. Chem., 91 (1987) 515.

[5] J.L. Figueiredo, M.F.R. Pereira, M.M.A. Freitas and J.J.M. Órfão; Carbon 37 (1999) 1379.

[6] J.L. Figueiredo, M.F.R. Pereira, M.M.A. Freitas and J.J.M. Órfão; Ind. Eng. Chem. Res., 46 (2007) 4110.

[7] K.S.W. Sing, D.H. Everett, R.A.W. Haul, L. Moscou, R.A. Pierotti, J. Rouquerol, T. Siemieniewska; Pure \& Appl. Chem., 57 (1985) 603.

\section{Acknowledgments}

BFM acknowledges FCT the PhD grant SFRH/BD/16565/2004. This work was supported by FCT and FEDER (projects POCTI/1181 and POCI/EQU/58252/2004). 